\title{
Advantages of real-time transabdominal ultrasound guidance in combined interstitial/intracavitary cervical brachytherapy: a case-based review
}

\author{
Ryan Thibodeau, MPH', Brittany A. Simone, DO', Sean Tanny, PhD², Seung Shin Hahn, MD', Paul D. Aridgides, MD' \\ 'Department of Radiation Oncology, SUNY Upstate Medical University, Syracuse, NY, USA, ${ }^{2}$ Department of Radiation Oncology, University \\ of Rochester Medical Center, Rochester, NY, USA
}

\begin{abstract}
Sub-optimal placement of both intracavitary devices and interstitial needles is a relatively common occurrence in cervical brachytherapy, which may reduce the accuracy of dose distribution and contribute to adverse toxicities. To mitigate complications, improve target dose coverage, and verify proper device placement, implants may be placed under real-time image guidance. Traditionally, transrectal ultrasound has been used for needle guidance. However, we have utilized transabdominal ultrasound (TA-US) in our brachytherapy center. The purpose of this pictorial essay was to provide a pictorial description of TA-US technique, present a retrospective review of our preliminary outcomes adopting TA-US into routine practice, and to discuss the advantages of real-time ultrasound image guidance for placement of intrauterine tandem and interstitial needles.

J Contemp Brachytherapy 2021; 13, 2: 211-220 DOI: https://doi.org/10.5114/jcb.2021.105290
\end{abstract}

Key words: brachytherapy, cervical cancer, transabdominal ultrasound, dosimetry, interstitial/intracavitary needles, high-dose-rate.

\section{Purpose}

Image-guided brachytherapy for cervical cancer using magnetic resonance imaging (MRI) has been shown to improve target coverage and provide meaningful improvements in local control [1, 2]. The routine use of cross-sectional imaging with computed tomography (CT) and MRI has facilitated delivering radiation to visible cervix and residual tumor as opposed to point A. Further incorporation of interstitial needles allows treatment beyond the reach of traditional intracavitary implants [3,4]. The American Brachytherapy Society (ABS) recommends the use of interstitial needles as they have been associated with improved dose to target for patients with bulky tumors, lower vaginal involvement, narrow vaginal apex, obliteration of the cervical os, pelvic sidewall, and parametrial involvement [5].

In 2015, our institution initiated high-dose-rate (HDR) brachytherapy for cervical cancer, which prior had utilized low-dose-rate (LDR) to deliver brachytherapy services to a large geographic area. Imaging capabilities were improved to include MRI imaging of the applicator placement in 2016. Combined free-hand needle intracavitary/interstitial (IC/IS) brachytherapy implants were initiated in 2017. While accurately placed free-hand IS needles improved target dose coverage, we proceeded cautiously to avoid puncture of nearby anatomical organs. To reduce this risk as well as improve confidence that distal needle position is within the target, our institution implemented transabdominal ultrasound (TA-US) guidance for tandem and needle placement in 2018. An added benefit from TA-US was the ability to perform treatments without the requirement for endocervical sleeves, which are prone to falling out during the course of treatment and may have unintended dosimetric effects $[6,7]$. In this pictorial essay, we provided a summary of the TA-US technique, presented clinical scenarios outlining the merits of real-time ultrasound guidance for IC/IS cervical brachytherapy, and reviewed dosimetric outcomes in the initial ten IC/IS brachytherapy patients treated at our center. The aim of this publication was to provide an overview of the benefits and limitations of TA-US for brachytherapy centers seeking to implement combined interstitial needle techniques with traditional intracavitary implants. In this regard, we have chosen representative image-based clinical scenarios and discussed this technique in relation to the existing literature supporting incorporation of real-time ultrasound with cervical brachytherapy. 


\section{Material and methods}

\section{Description of real-time transabdominal ultrasound-guided cervical brachytherapy}

All cases of definitive radiation for cervical cancer undergo multidisciplinary review by a gynecology oncology and radiation oncology team with brachytherapy expertise prior to initiating treatment. Patients are re-evaluated with clinical examination on the final week of external beam radiation therapy (EBRT) to ensure the cervical os is visible for tandem insertion. An MRI is obtained prior to implantation for pre-procedure planning of bulky tumors, particularly to plan needle use with regard to number, location, and depth of insertion. Brachytherapy is delivered using a titanium MRI-compatible flexible geometry Fletcher suit device (Varian Medical Systems, Palo Alto, CA, USA) and flexible plastic interstitial needles.
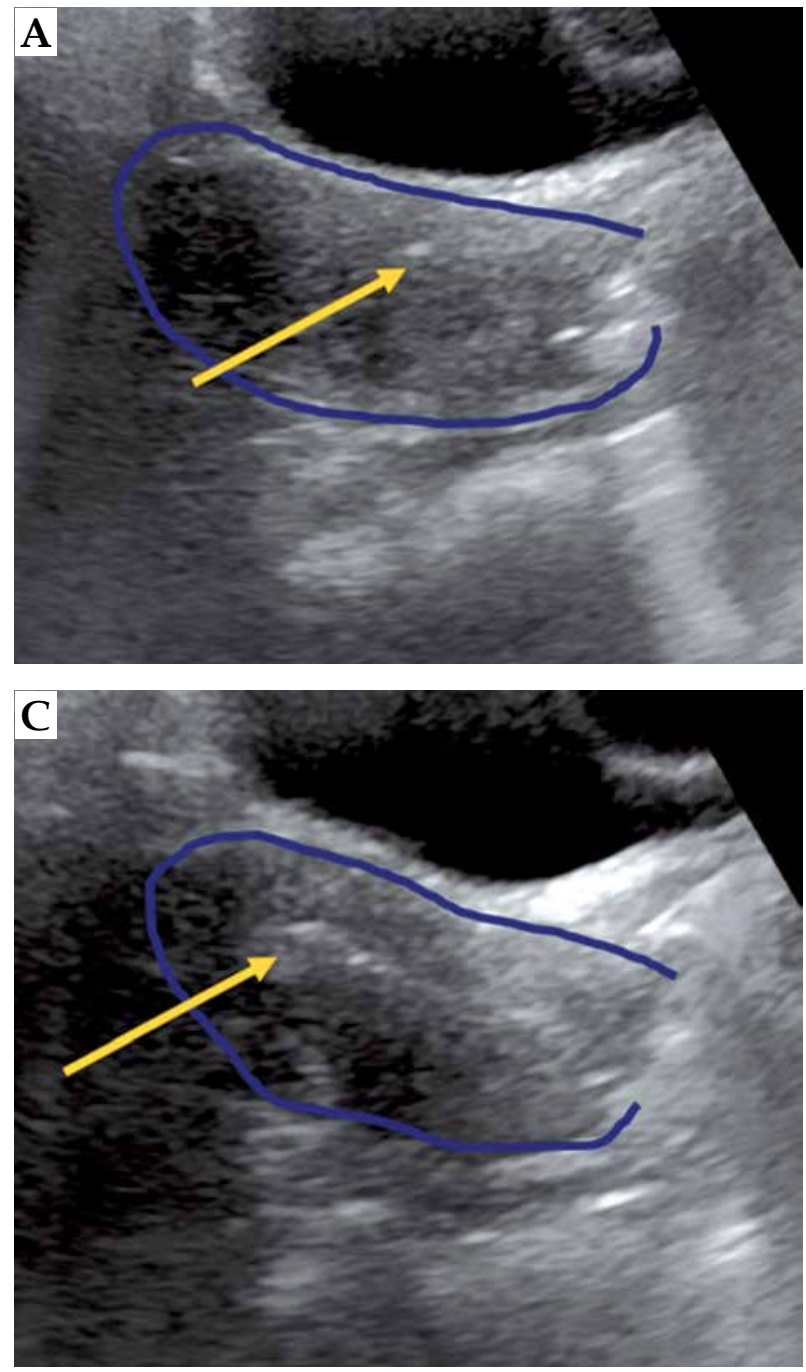

The patient is prepped in sterile fashion and placed in the lithotomy position under monitored anesthesia care or general anesthesia. Using a metal speculum, the cervical os is identified, dilated, and then the intrauterine canal is sounded for depth with TA-US guidance. If there is inadequate visualization due to bowel image distortion or large body habitus, normal saline is instilled into the bladder through the foley catheter. The tandem is then placed under TA-US guidance to ensure the tandem reaches the uterine fundus, with representative pictures showing the tandem initially within the cervix (Figure 1A), advancing along the intrauterine canal (Figure 1B, C), and ultimately reaching the uterine fundus (Figure 1D). Following adequate placement of the intrauterine tandem (Figure 2A), the tandem is then manipulated in all directions to determine the location of needles, which can be either superior, inferior, or lateral to the tandem as well as medial or lateral to the planned ovoid locations. A metal stylet is
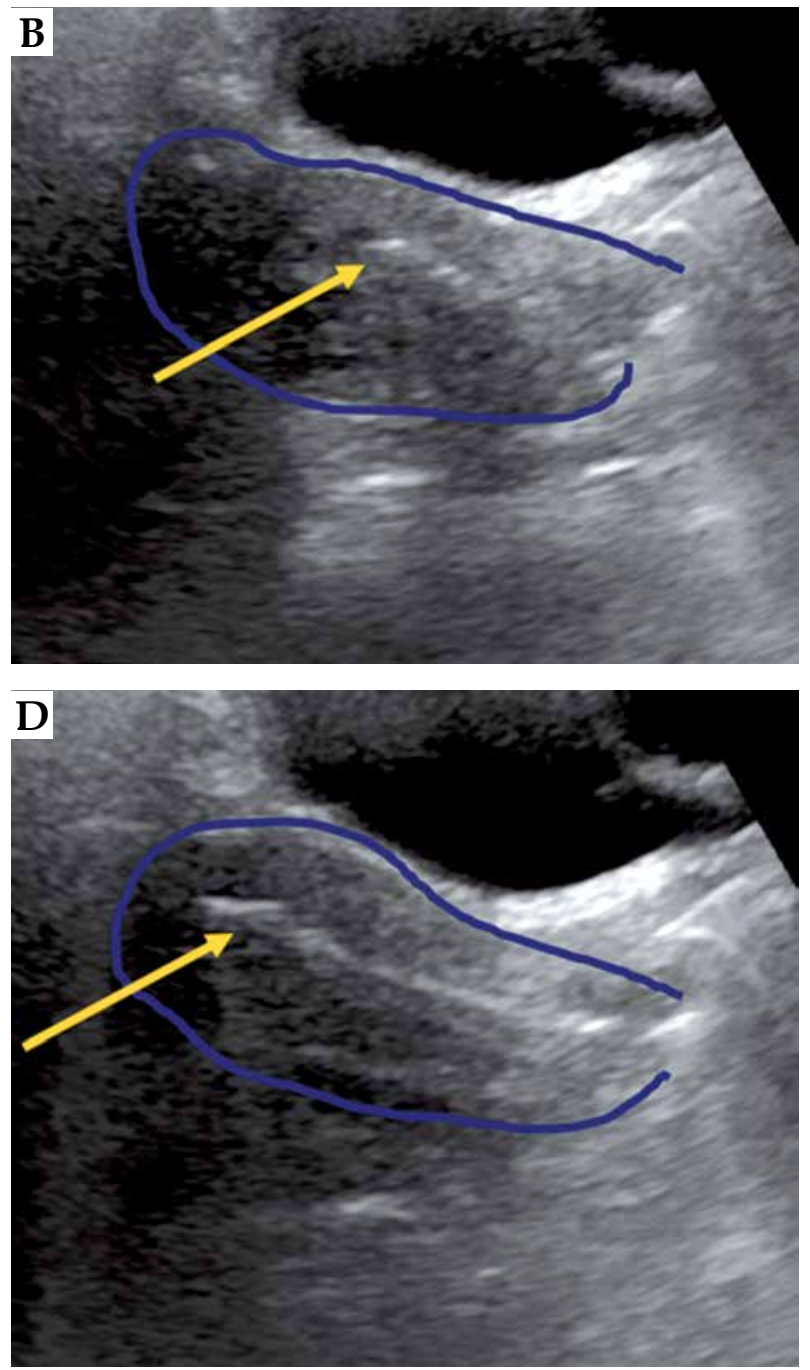

Fig. 1. Representative patient with stage IIB cervical cancer illustrating placement of the intrauterine tandem (yellow arrow) into the cervix (A), advancing further within the endometrial cavity (B and $\mathbf{C})$, and final location of the tandem tip at the uterine fundus (D). For this patient, there was significant resistance noted in the cervix $1 \mathrm{~cm}$ past the cervical os, and transabdominal ultrasound (TA-US) guidance provided reassurance that application of careful force in the correct location and direction facilitated successful placement 
utilized for needle placement and visualization under TAUS guidance to limit advancement beyond the cervix and uterus (Figure 2B, C). All patients undergo post-implant pelvic CT for brachytherapy planning (Figure 2D). Pelvic MRI is performed with the implant in place for 1-3 of 5 total brachytherapy fractions (as early in brachytherapy course as can be obtained) to better define target volume and nearby normal organs. The value of TA-US is particularly evident for cases where on the first fraction, the tandem was not well visualized in the uterus (Figure 3A), and MRI imaging confirmed sub-optimal placement (Figure $3 \mathrm{~B}$ ). While the challenging nature of this case is due to very extensive residual cervical tumor, TA-US guidance is successful for tandem placement (Figure 3C) for her subsequent fractions with MRI (fraction 4), highlighting significant tumor response (Figure 3D).

To provide context of studies supporting the use of real-time image guidance with cervical brachytherapy, a literature search from years 1990-2020 was performed in MEDLINE and PubMed for cervical brachytherapy that included image guidance, needle placement, implant dosimetry, and treatment toxicity.
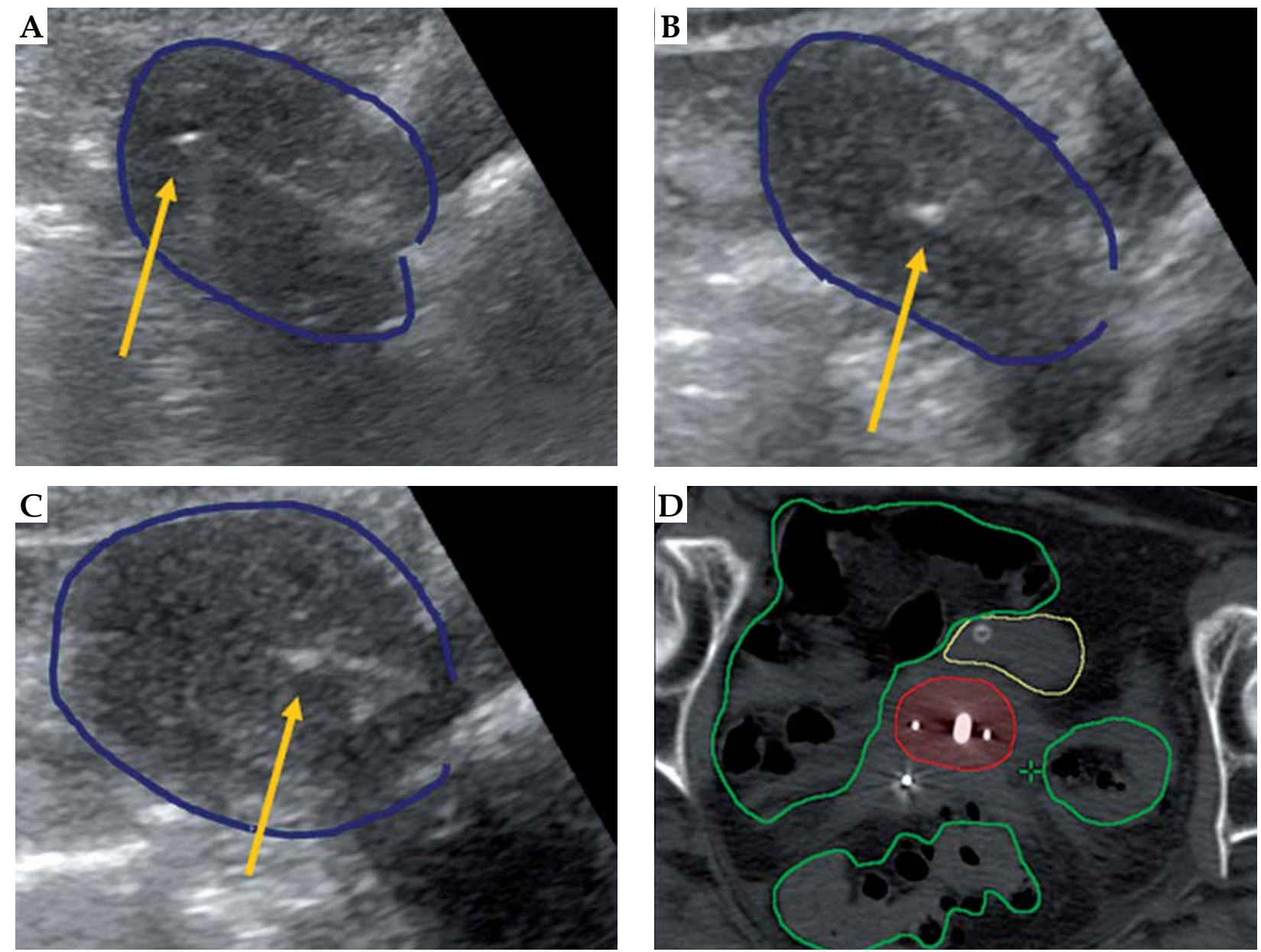

Fig. 2. Representative patient with stage IIB cervical cancer receiving combined intracavitary/interstitial brachytherapy with transabdominal ultrasound (TA-US) guidance. TA-US images for tandem placement (A), right interstitial needle (B), and left interstitial needle (C) are shown with the uterus (dark blue). An axial view of the CT scan for brachytherapy planning (D) confirms position of the tandem with two lateral needles to the right and left, with contours for the high-risk clinical target volume (red), bowel (green), and bladder (yellow) also shown 

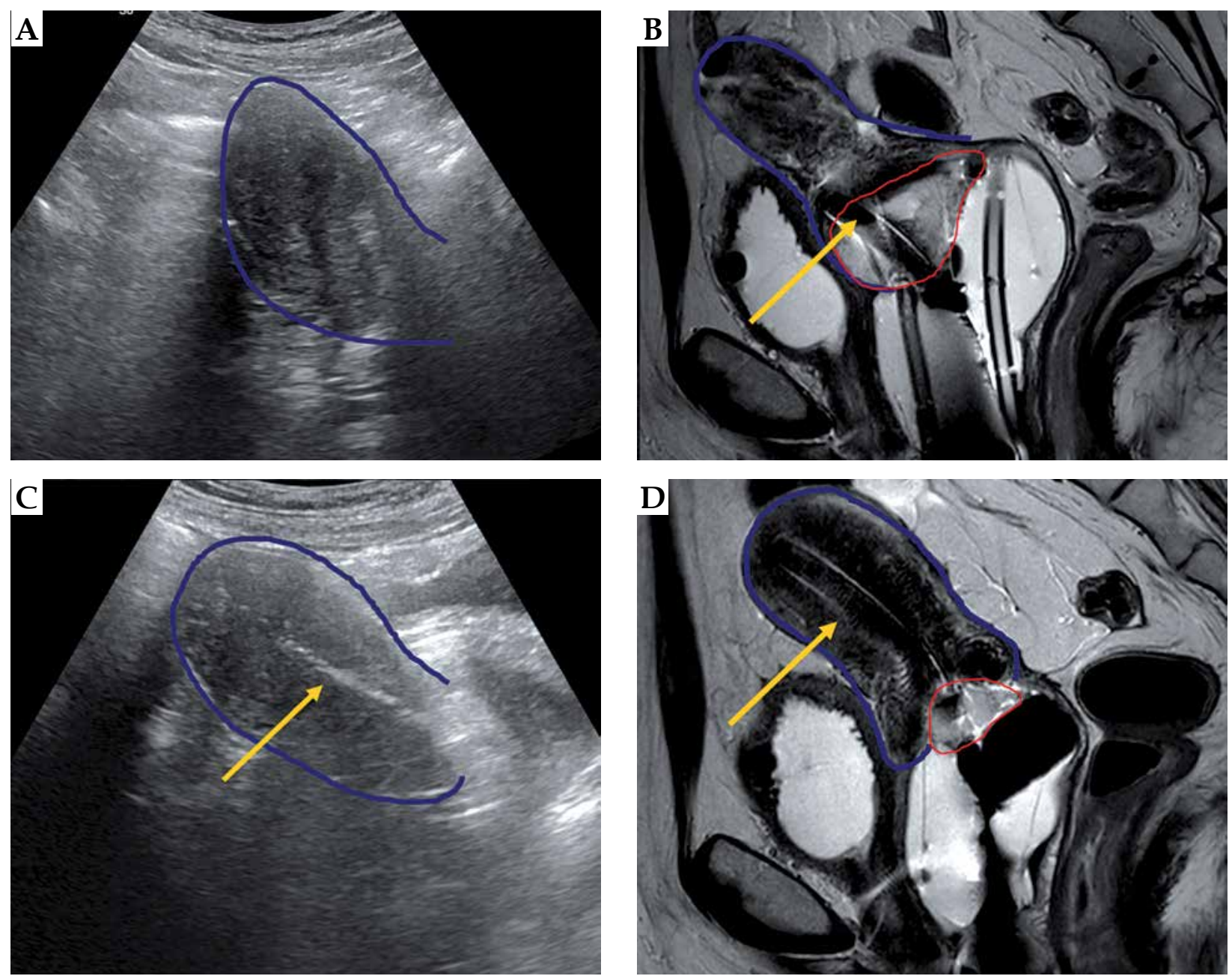

Fig. 3. Representative patient with cervical adenocarcinoma, stage IB $6 \mathrm{~cm}$ tumor, where on the first fraction of brachytherapy, the intrauterine tandem was not visualized (A) and resulted in inappropriate location (B). On subsequent brachytherapy fractions, with fraction 4 illustrated above (C and $\mathbf{D})$, adequate intrauterine tandem placement was achieved, and the extensive residual cervical tumor evidenced at the start of brachytherapy (B) exhibited a significant regression (D)

siderations. These patients were treated with fourfield three-dimensional conformal radiation therapy (3D-CRT), as this was standard practice at those facilities at the time of treatment. EBRT included standard primary, pelvic nodal, and selective para-aortic nodal coverage at the discretion of treating physician. Four patients were treated with 3D-CRT. Six were treated with IMRT, of which three utilized simultaneous integrated boost to gross nodes (median total dose, $55 \mathrm{~Gy}$; 2.05 Gy per fraction). Patients receiving EBRT at SUNY Upstate were treated with either Varian TrueBeam or Accuray TomoTherapy. Treatment planning for EBRT was completed using Varian Eclipse (Varian Medical Systems, Palo Alto, CA, USA).

Brachytherapy was delivered using a titanium MRI-compatible flexible geometry Fletcher suit device and flexible plastic interstitial needles. Brachytherapy boost was delivered as 30 Gy in 5 fractions, to ensure at least $90 \%$ of high-risk clinical target volume (HR-CTV) received prescription, unless rectum, bladder, or bowel planning aims could not be met. All patients underwent post-implant pelvic CT for brachytherapy plan- ning. Treatment factors and toxicity analyzed included: real-time image guidance with TA-US, early toxicities of treatment with attention to grade $\geq 3$ toxicities, tumor stage, planning imaging modality (CT vs. MRI), dosimetric parameters, and chemotherapy. Pelvic MRI was performed prior to either the first or second fraction of brachytherapy (in addition to pelvic CT scan) to better define target volume and nearby normal organs. TA-US was used to guide proper insertions of tandem and interstitial needles in the majority of fractions for the last 6/10 patients. Varian BrachyVision (Varian Medical Systems, Palo Alto, CA, USA) was used for brachytherapy planning and for gathering dosimetric data. Statistical analyses (two-tailed $t$-test) comparing dosimetric parameters for fractions with or without TA-US guidance were conducted using IBM Statistical Package for the Social Sciences Software (version 25; IBM, Armonk, NY, USA). Kolmogorov-Smirnov normality testing yielded normality $(p>0.05)$ for HR-CTV $D_{90}$, rectal $\mathrm{D}_{2 \mathrm{cc}}$, bladder $\mathrm{D}_{2 \mathrm{cc}}$ and bowel $\mathrm{D}_{2 \mathrm{cc}}$. 


\section{Advantages of TA-US cervical brachytherapy}

\section{Improved dosimetry of brachytherapy plans}

Clinical and dosimetric data for patients included in this preliminary review are shown in Table 1 [8]. With a mean patient follow-up of 13.1 months (median, 7.6 months), fractions using transabdominal ultrasound (TA-US) when compared to fractions without TA-US were found to have an increased HR-CTV $\mathrm{D}_{90}(6.54 \mathrm{~Gy}$ vs. $5.95 \mathrm{~Gy}, p<0.01)$ and decreased dose to $2 \mathrm{cc}\left(\mathrm{D}_{2 \mathrm{cc}}\right)$ rectum (2.63 Gy vs. $3.02 \mathrm{~Gy}, p<0.01)$. $\mathrm{D}_{2 \mathrm{cc}}$ of the bladder and bowel/sigmoid were not significant. Similarly, the average $\mathrm{D}_{2 \mathrm{cc}}$ rectum for patients with 3 or more US fractions was 2.49 Gy compared to $3.27 \mathrm{~Gy}$, with 2 or fewer US fractions.

\section{Placement of intrauterine tandem}

Placement of the intrauterine tandem in cases of locally advanced cervical cancer is often challenging due to ulcerative or infiltrative tumor as well as post-radiation changes. As a result, sub-optimal placement resulting in malposition may lead to uterine perforation or malposition [9]. Patient 4 (Table 1) is an example of suspected uterine perforation as a result of tandem placement without visual confirmation from TA-US (Figure 4A, B). The suspected perforation was not immediately evident at the time of brachytherapy delivery, as the tandem tip was noted to be in close proximity to the uterine wall but not overtly penetrating. As a modification to the typical brachytherapy delivery, the most distal $2 \mathrm{~cm}$ tandem positions were not used with source dwell times; although in retrospect, removal and repositioning of the implant would be our preference. For the patient's remaining fractions, the tandem placement was confirmed in real-time with TA-US guidance (Figure 4C, D). This patient unfortunately developed small bowel obstruction in close proximity to the site of possible uterine perforation, one month following brachytherapy. She had a successful diverting colostomy but ultimately expired with evidence of active/residual disease three months after the treatment completion. For the subsequent six patients (30 fractions of HDR brachytherapy) treated with combined IC/IS techniques, we found no cases of intrauterine rupture with TA-US used in 28 fractions $(93 \%)$, and have since incorporated TA-US into routine practice.

\section{Placement of interstitial needles}

The incorporation of interstitial needles to intracavitary implants is associated with superior local control in bulky tumors (HR-CTV $\geq 30 \mathrm{~cm}^{3}$ ) and improvements in average dose (HR-CTV) across all tumor sizes [10]. Although perineal interstitial implants leading to visceral organ puncture appear to be safe (provided sources are not loaded in distal positions), there is still some reluctance among brachytherapy practitioners to adopt combined interstitial brachytherapy techniques [11-13]. In our center, we adopted TA-US within one year of initiating IS/IC implants. Figure 5 shows a recently treated patient at our institution, in whom TA-US allowed careful placement of posterior interstitial needles to residual tumor

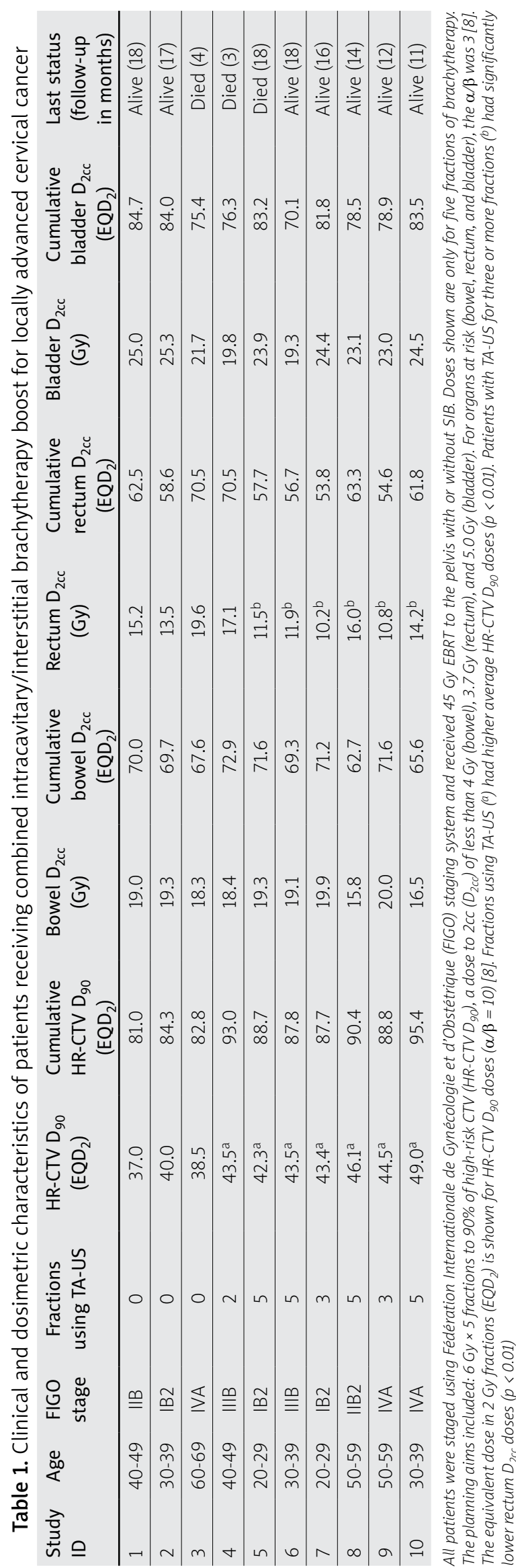



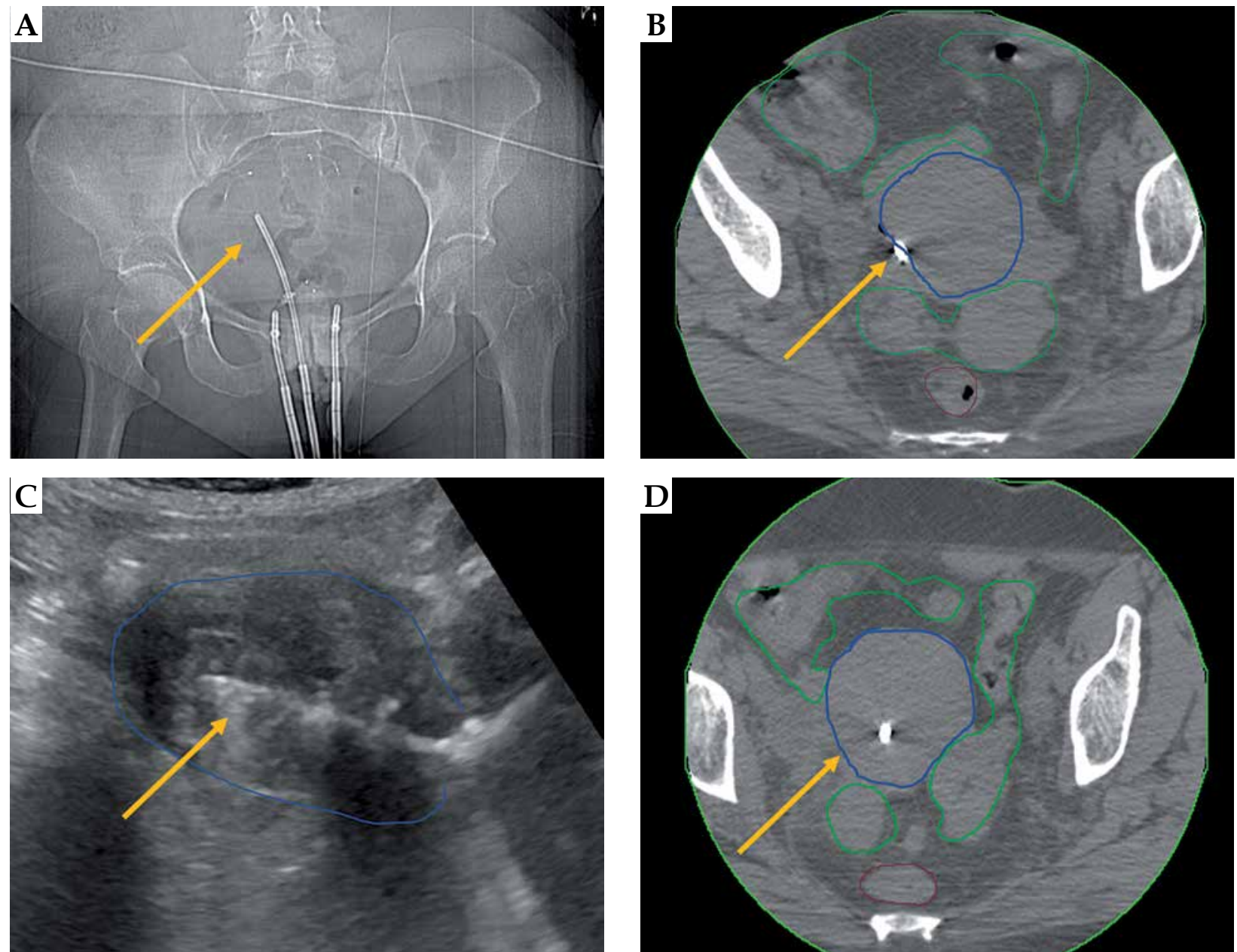

Fig. 4. Representative patient with stage IIIB cervical cancer, illustrating benefit of transabdominal ultrasound (TA-US) guidance with intrauterine tandem placement. X-ray (A) and CT images (B) show tandem tip (yellow arrow) in close proximity to the uterine wall (dark blue). Due to lateral position of the tandem tip, the most distal $2 \mathrm{~cm}$ dwell positions were not loaded. In retrospect, the placement may resulted in uterine perforation as a possible contribution to the patient developing a small bowel obstruction 1 month from brachytherapy. For the subsequent fractions with TA-US, the optimal tandem placement was confirmed (C) prior to CT scanning (D) and brachytherapy delivery. The bowel (green) and rectum (brown) are contoured

seen on post-EBRT MRI, with avoidance of sigmoid or rectal puncture. As an additional example, real-time TA-US guidance was utilized for the placement of two anterior needles in a patient with bladder involvement (Figure 6). This patient remains disease-free two years following completion of IS/IC brachytherapy. TA-US can be directed to the right and left aspects of cervical tumor for bilateral parametrial/paracervical needle implantations as well as distinguish anterior and posterior needle locations simultaneously (Figure 7).

\section{Discussion}

The Groupe Européen de Curiethérapie (GEC) and the European Society for Radiotherapy and Oncology (ESTRO) had recognized the use of ultrasonography as a valuable tool in image-assisted treatment planning, application guidance, and definitive treatment planning [14]. Similarly, the Indian Brachytherapy Society guidelines recommends the use of either transrectal ultrasound
(TRUS) or rectal exam to guide advancement of interstitial needles [15]. While this is consistent with 2012 ABS consensus recommendations, only $56 \%$ of ABS members reported using ultrasound for cervical brachytherapy in a 2010 survey [16, 17]. In our institutional experience, routine use of TA-US was an integral component of a several year transition from low-dose-rate brachytherapy prescribing to point A to imaged-guided brachytherapy combining interstitial and intracavitary applications, particularly to improve accuracy with needle placement. An unexpected finding from the dosimetry review of our initial 10 patients treated with IC/IS brachytherapy found that TA-US was associated with improved tumor coverage (HR-CTV $\mathrm{D}_{90}$ ) as well as lower rectal dose (rectum, $\mathrm{D}_{2 \mathrm{cc}}$ ). This analysis was preliminary only and has several limitations, including small patients' number and increasing brachytherapist experience with IC/IS over time that also coincided with the use of TA-US. These biases and small patients' number are significant and may had contributed to evidenced dosimetric improvements. As 

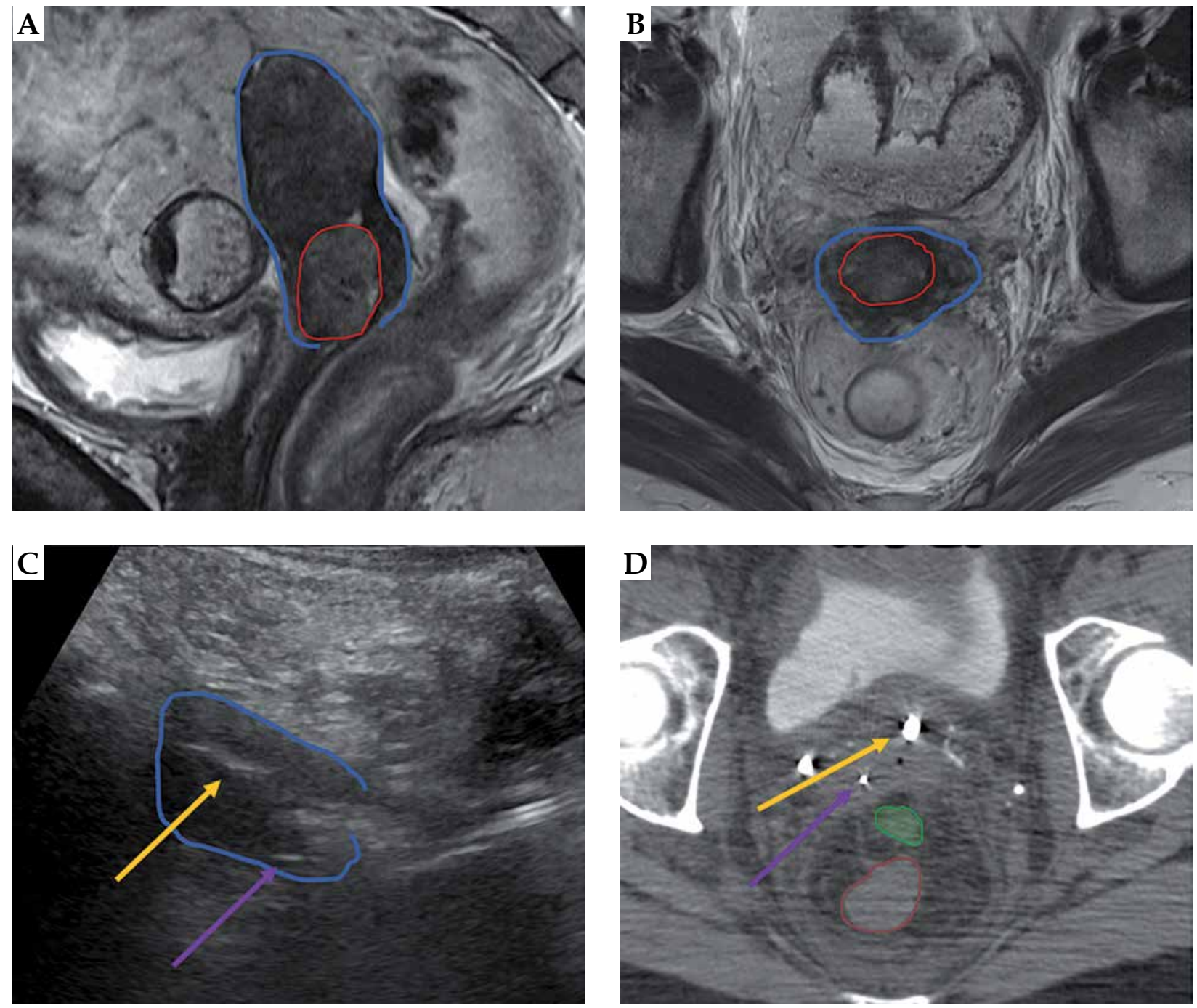

Fig. 5. Representative patient with gross residual tumor. Pre-implantation MRI in the sagittal (A) and axial (B) orientations show gross residual tumor (red) in relation to the uterus (blue). The patient had a placement of tandem (yellow arrow) and right posterior interstitial needle (purple arrow) with transabdominal ultrasound (TA-US) guidance (C). Brachytherapy planning CT scan (D) confirmed favorable needle location relative to tandem and residual tumor, with bowel (green) and rectum (brown) also shown. In $\mathbf{D}$, a right ureteral stent is just anterior to the midpoint of yellow arrow (stage IIIB disease)

our providers had since universally adopted TA-US for cervical brachytherapy, no further patients treated without the use of this modality would be available for study at our center.

Sub-optimal placement of intracavitary applicators has been shown to occur in up to $32 \%$ of cases of cervical brachytherapy, with a uterine perforation risk of $\sim 5-10 \%$ in studies without using ultrasound guidance $[9,18,19]$. In a retrospective study from London Health Sciences Center in London, Ontario, incorporation of TA-US was shown to decrease the rate of uterine perforation (from $10 \%$ to $3 \%$ ) as well as decrease both procedure time and likelihood to consult gynecology oncologist assistance (from $38 \%$ to $5.7 \%$ ) [19]. Investigators at Massachusetts General Hospital did not find any cases of uterine perforation for 73 consecutive cases following adoption of TA-US compared to $10 \%$ of cases prior to TA-US use [18]. In similar fashion, the prospective use of TA-US in
96 patients at the University of Alabama without a visible cervical os resulted in successful cervical tandem passage in 94 cases with no uterine perforation [20]. It is important to note, that particularly in high-volume centers with experienced brachytherapists, the rate of perforation and inadequate placement may be less than reported in the literature and therefore, the incorporation of TA-US could be less impactful. The imaging limitations we have encountered included diminished visualization of targets in patients with large body habitus and/or presence of significant bowel loops in the pelvis. The routine use of TA-US has not added significantly to procedure time at our institution, as the ultrasound equipment and technologists are readily available; although, we envision these could be potential barriers to implementation at other centers.

Previous studies have shown that TRUS, especially when combined with CT for dose optimization, provides 

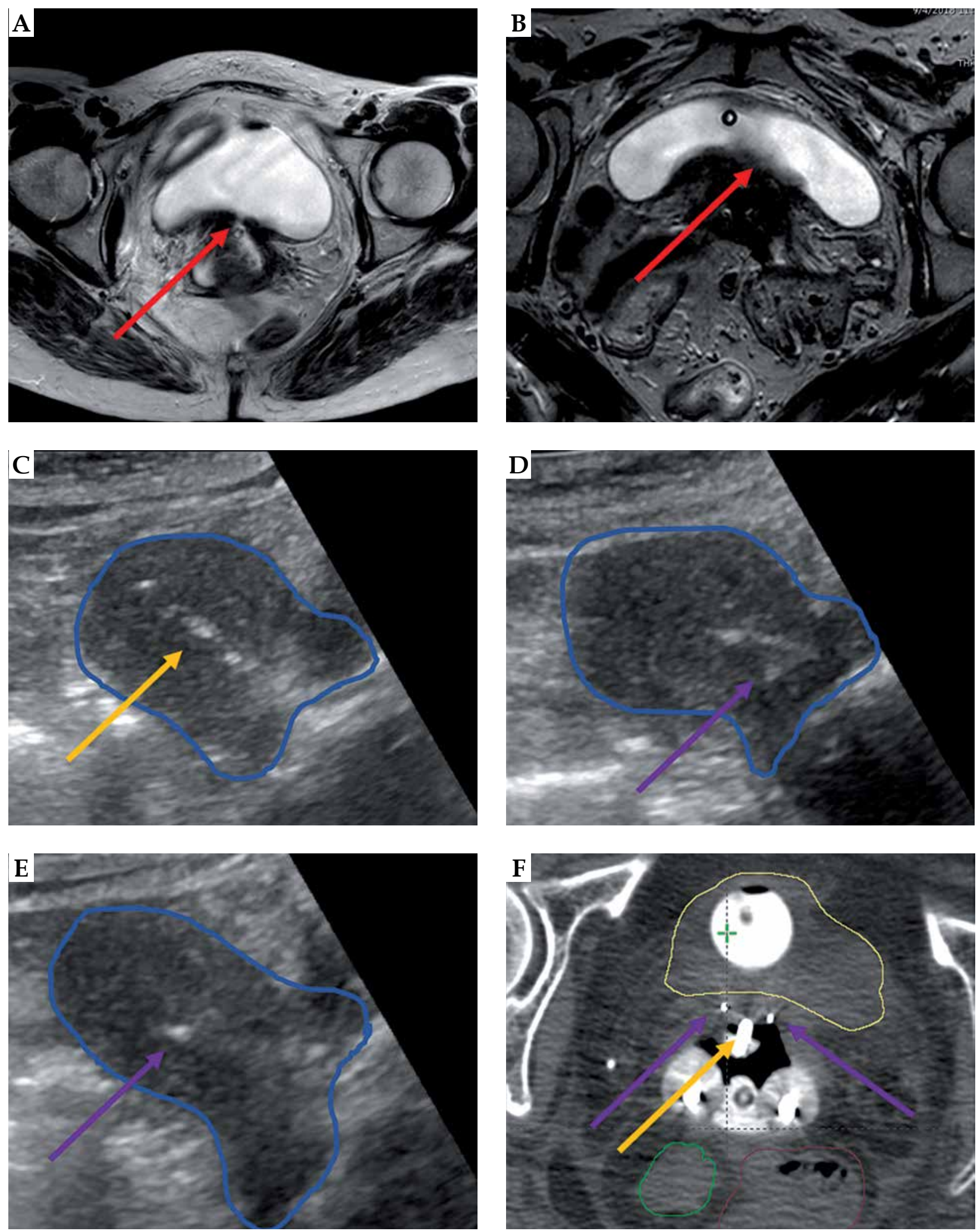

Fig. 6. Representative patient with bladder invasion, stage IVA, cervical cancer resulting in vesicovaginal fistula. At diagnosis (A), bladder invasion (red arrow) was seen on MRI. On pre-implantation MRI (B), this invasion appeared to result in vesicovaginal fistula (red arrow). The patient had a placement of tandem (yellow arrow) and two anterior interstitial needles (purple arrow) with transabdominal ultrasound (TA-US) guidance (C-E). The uterus contour is shown in blue. Brachytherapy planning CT scan (F) confirmed favorable needle locations relative to residual tumor and bladder (yellow) with intracavitary ovoids and posterior vaginal balloon posteriorly. The bowel (green) and rectum (brown) are also contoured 

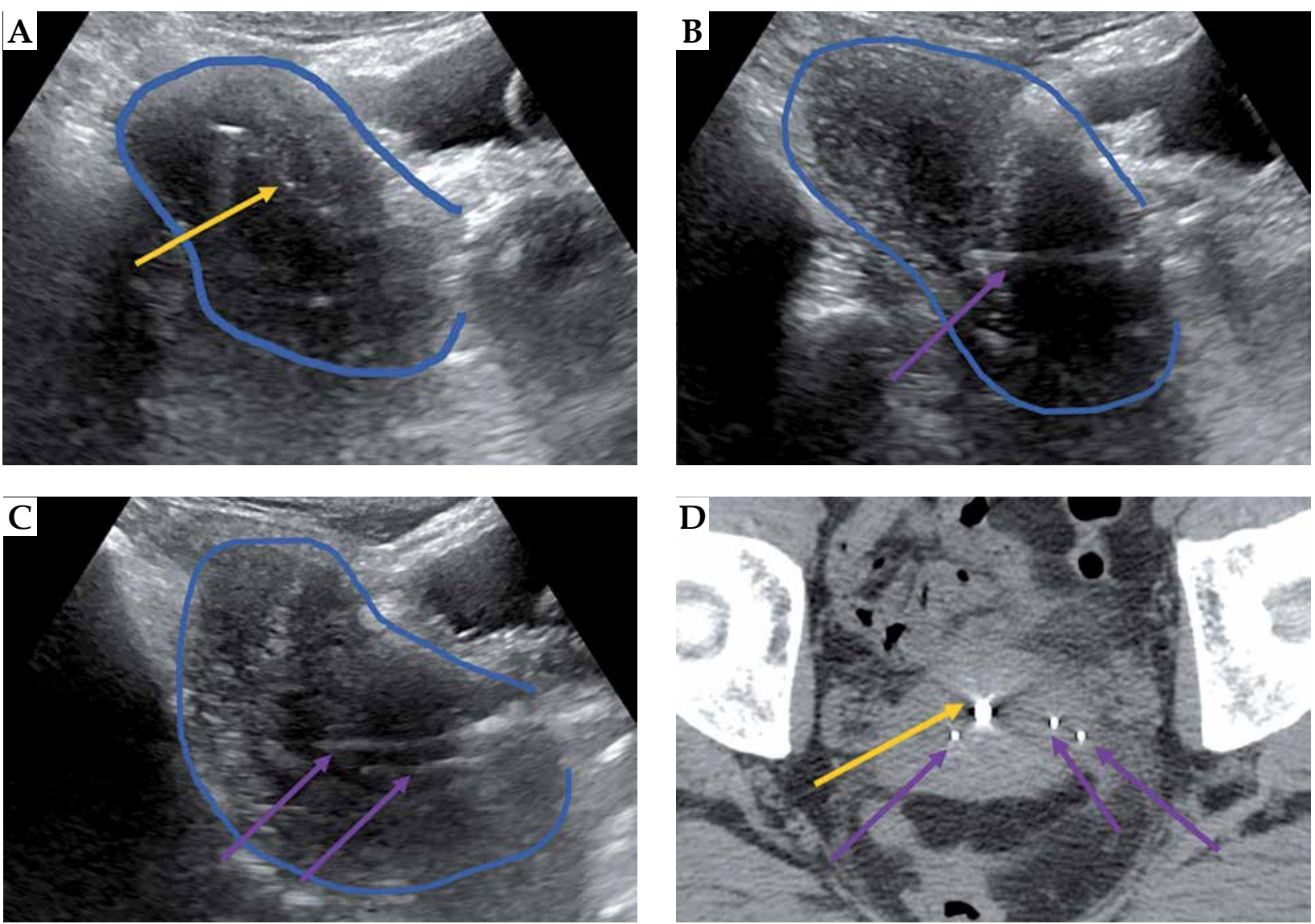

Fig. 7. Representative patient with IB cervical cancer, $5 \mathrm{~cm}$ tumor, with residual tumor at the time of combined intracavitary (A) and interstitial (B-D) brachytherapy. Transabdominal ultrasound (TA-US) guidance aided with tandem placement (yellow arrow) as well as interstitial needles (purple arrows) on the right (B) and left (C) tumor locations

clinically comparable results to MRI-based treatment planning [21, 22]. In a proof-of-concept study utilizing combined TRUS and MRI for IC/IS in cervical cancer, TRUS images were reported to confirm needle tip position as well as adequate alignment of the tandem and ring applicator [22]. However, the potential limitations of TRUS included a small field of view that precluded full visibility of the applicator and organs at risk, and movement of the ring applicator that occurred during withdrawal of the transducer. Investigators from the University of Western Ontario confirmed TRUS to be useful for verification of needle placement [23]. Of 5 patients treated with 73 total needles, 64 needles $(88 \%)$ were visualized on TRUS including 58 (79\%) where the needle tip was identified. Most cases of inadequate visualization were due to shadowing from the vaginal cylinder of needle template, which would be avoided with free-hand techniques. The authors concluded that the remaining cases of poor needle tip visibility resulted from artifact from other needles and fecal matter remaining in the rectum. In a study of template-based interstitial brachytherapy for gynecologic malignancies that included four patients with cervical cancer, TRUS was successfully used for needle insertion in the transverse plane, and then advanced to appropriate depth using longitudinal imaging. The bladder and rectum were directly imaged and could be avoided from puncture [24].
While needle visualization is reported to be superior with TRUS as compared to TA-US, interstitial needle placement with TA-US guidance has been relatively investigated $[14,25]$. Potential advantages of TA-US we identified in our brachytherapy center include ultrasound technologist support for image acquisition that can work from outside of the limited lithotomy position physical arena, lack of ultrasound hardware in the brachytherapy field, and no requirements for bowel preparation. Furthermore, TA-US was convenient, immediately available, and cost-effective [26]. As our brachytherapy center incorporated real-time MRI for image guidance, the benefit of interstitial needle placement into residual gross tumor volume was readily appreciated. The routine use of TAUS increased brachytherapist comfort with interstitial needle placement as well as intrauterine tandem insertion without requiring endocervical sleeves. We found that the incorporation of TA-US as a preliminary finding also improved HR-CTV and rectal doses for patients receiving IC/IS cervical brachytherapy. We hypothesize that it may be a useful technology for other brachytherapy centers, which are starting to adopt IC/IS techniques and for practitioners without TRUS availability or expertise. One of the upcoming major aims of our brachytherapy center is to gain experience with TRUS for further clinical comparisons to our outcomes with TA-US. 
As TA-US had preliminarily allowed us to increase HRCTV $\mathrm{D}_{90}$ dose without increasing dose to organs at risk, as being evaluated in the EMBRACE II study, we plan to continue this practice for patients with large burden of disease at the time of brachytherapy. Future studies will continue to examine both patient outcomes and impact of real-time image guidance with adoption of this strategy [27].

All research was conducted at State University of New York (SUNY) Upstate Medical University, Department of Radiation Oncology, 750 East Adams Street, Syracuse, New York, 13210.

\section{Disclosure}

The authors report no conflict of interest.

\section{References}

1. Kirisits C, Lang S, Dimopoulos et al. The Vienna applicator for combined intracavitary and interstitial brachytherapy of cervical cancer: design, application, treatment planning, and dosimetric results. Int J Radiat Oncol Biol Phys 2006; 65: 624630.

2. Mazeron R, Castelnau-Marchand P, Escande A et al. Tumor dose-volume response in image-guided adaptive brachytherapy for cervical cancer: A meta-regression analysis. Brachytherapy 2016; 15: 537-542.

3. Viswanathan AN, Erickson B, Gaffney DK et al. Comparison and consensus guidelines for delineation of clinical target volume for CT- and MR-based brachytherapy in locally advanced cervical cancer. Int J Radiat Oncol Biol Phys 2014; 90: 320-328.

4. Ohno T, Wakatsuki M, Toita T et al. Recommendations for high-risk clinical target volume definition with computed tomography for three-dimensional image-guided brachytherapy in cervical cancer patients. J Radiat Res 2017; 58: 341-350.

5. Viswanathan AN, Beriwal S, De Los Santos JF et al. American Brachytherapy Society consensus guidelines for locally advanced carcinoma of the cervix. Part II: high-dose-rate brachytherapy. Brachytherapy 2012; 11: 47-52.

6. Jan YT, Chang CL, Tai HC et al. The air matters - sleeve air cavity as a marker guiding image-guided helical tomotherapy to target cervical cancer. J Contemp Brachytherapy 2016; 8: 82-87.

7. Mehta S, Farnia B, de la Zerda A et al. Dosimetric effects of the Smit sleeve on high-dose-rate brachytherapy tandem and ovoids plans for patients with locally advanced cervical cancer. J Contemp Brachytherapy 2019; 11: 584-588.

8. Dimopoulos JC, Lang S, Kirisits C et al. Dose-volume histogram parameters and local tumor control in magnetic resonance image-guided cervical cancer brachytherapy. Int $\mathrm{J} R \mathrm{a}$ diat Oncol Biol Phys 2009; 75: 56-63.

9. Kissel M, Silva M, Lequesne J et al. Impact of suboptimal tandem implantation on local control and complications in intracavitary brachytherapy for cervix cancer. Brachytherapy 2019; 18: 753-762.

10. Fokdal L, Sturdza A, Mazeron R et al. Image guided adaptive brachytherapy with combined intracavitary and interstitial technique improves the therapeutic ratio in locally advanced cervical cancer: Analysis from the retroEMBRACE study. Radiother Oncol 2016; 120: 434-440.

11. Shah AP, Strauss JB, Gielda BT et al. Toxicity associated with bowel or bladder puncture during gynecologic interstitial brachytherapy. Int J Radiat Oncol Biol Phys 2010; 77: 171-179.
12. Mendez LC, Lang P, Patel C et al. A prospective analysis of catheter complications for gynecological cancers treated with interstitial brachytherapy in the 3D era. Brachytherapy 2019; 18: $44-49$

13. Taggar AS, Phan T, Traptow L et al. Cervical cancer brachytherapy in Canada: A focus on interstitial brachytherapy utilization. Brachytherapy 2017; 16: 161-166.

14. Siebert FA, Kirisits C, Hellebust TP et al. GEC-ESTRO/ ACROP recommendations for quality assurance of ultrasound imaging in brachytherapy. Radiother Oncol 2020; 148: 51-56.

15. Mahantshetty U, Gudi S, Singh R et al. Indian Brachytherapy Society Guidelines for radiotherapeutic management of cervical cancer with special emphasis on high-dose-rate brachytherapy. J Contemp Brachytherapy 2019; 11: 293-306.

16. Viswanathan AN, Thomadsen B. American Brachytherapy Society consensus guidelines for locally advanced carcinoma of the cervix. Part I: general principles. Brachytherapy 2012; 11: 33-46.

17. Viswanathan AN, Erickson BA. Three-dimensional imaging in gynecologic brachytherapy: a survey of the American Brachytherapy Society. Int J Radiat Oncol Biol Phys 2010; 76: 104-109.

18. Granai CO, Doherty F, Allee P et al. Ultrasound for diagnosing and preventing malplacement of intrauterine tandems. Obstet Gynecol 1990; 75: 110-113.

19. Davidson MTM, Yuen J, D'Souza DP et al. Optimization of high-dose-rate cervix brachytherapy applicator placement: the benefits of intraoperative ultrasound guidance. Brachytherapy 2008; 7: 248-253.

20. Schaner PE, Caudell JJ, De Los Santos JF et al. Intraoperative ultrasound guidance during intracavitary brachytherapy applicator placement in cervical cancer: the University of Alabama at Birmingham experience. Int J Gynecol Cancer 2013; 23: 559-566.

21. Mahantshetty U, Naga ChP, Khadanga CR et al. A prospective comparison of computed tomography with transrectal ultrasonography assistance and magnetic resonance imaging-based target-volume definition during image guided adaptive brachytherapy for cervical cancers. Int J Radiat Oncol Biol Phys 2018; 102: 1448-1456.

22. Nesvacil N, Schmid MP, Potter R et al. Combining transrectal ultrasound and CT for image-guided adaptive brachytherapy of cervical cancer: Proof of concept. Brachytherapy 2016; 15: 839-844.

23. Rodgers JR, Surry K, Leung E et al. Toward a 3D transrectal ultrasound system for verification of needle placement during high-dose-rate interstitial gynecologic brachytherapy. Med Phys 2017; 44: 1899-1911.

24. Stock RG, Chan K, Terk M et al. A new technique for performing Syed-Neblett template interstitial implants for gynecologic malignancies using transrectal-ultrasound guidance. Int J Radiat Oncol Biol Phys 1997; 37: 819-825.

25. Kamrava M. Potential role of ultrasound imaging in interstitial image based cervical cancer brachytherapy. J Contemp Brachytherapy 2014; 6: 223-230.

26. Rao PB, Ghosh S. Routine use of ultrasound guided tandem placement in intracavitary brachytherapy for the treatment of cervical cancer - a South Indian institutional experience. J Contemp Brachytherapy 2015; 7: 352-356.

27. Pötter R, Tanderup K, Kirisits C et al. The EMBRACE II study: The outcome and prospect of two decades of evolution within the GEC-ESTRO GYN working group and the EMBRACE studies. Clin Transl Radiat Oncol 2018; 9: 48-60. 